\title{
Pancreatitis autoinmune en pediatría. Caso clínico
} Autoimmune pancreatitis in children. Clinical case

Dra. Ma. Alejandra Mortarini, Dra. Johana Hincapie y Dra. Ana Rocca ${ }^{a}$

\section{RESUMEN}

La pancreatitis recurrente ocurre en el $15-35 \%$ en la edad pediátrica. Se define como 2 o más episodios distintos de pancreatitis aguda con normalización de enzimas pancreáticas entre cada episodio. Una de sus causas es la pancreatitis autoinmune. En los últimos 10 años se controlaron, en el Hospital Garrahan, 10 pacientes con diagnóstico de pancreatitis recurrente, de los cuales solo uno tuvo diagnóstico de pancreatitis autoinmune. Se describe el caso clínico de una paciente, que, inicialmente, tenía estudios normales de función y anatomía pancreática y, en la evolución, luego de un episodio de pancreatitis aguda, desarrolló estenosis del conducto de Wirsung sugestiva de pancreatitis autoinmune. Se considera importante describir esta patología infrecuente en pediatría, pero que se encuentra en auge.

Palabras clave: pancreatitis, pancreatitis crónica, pancreatitis aguda necrotizante.

\section{ABSTRACT \\ Introduction. Recurrent pancreatitis occurs in children between 15 and $35 \%$ of the cases. It is defined as two or more separate episodes of acute pancreatitis with normalization of the pancreatic enzymes between episodes. One of the causes is autoimmune pancreatitis. Over the last 10 years, 10 patients with recurrent pancreatitis were sent at our center. Only one was considered to have autoimmune pancreatitis. We described a clinical case about a patient, who had, at the beginning, normal functional and anatomical studies, and then was finally diagnosed with autoimmune disease based on findings on the magnetic resonance cholangiopancreatography with a duct of Wirsung abnormality. We considered important to describe this uncommon disorder in childhood, in spite of having an increasing incidence. \\ Key words: pancreatitis, chronic pancreatitis, acute necrotizing pancreatitis.}

http: / / dx.doi.org/10.5546/ aap.2021.e229

Cómo citar: Mortarini MA, Hincapie J, Rocca A. Pancreatitis autoinmune en pediatría. Caso clínico. Arch Argent Pediatr 2021;119(3):e229-e233.

a. Servicio de Gastroenterología, Hospital de Pediatría SAMIC "Prof. Dr. Juan P. Garrahan", Ciudad Autónoma de Buenos Aires, Argentina.

Correspondencia:

Dra. Ma. Alejandra Mortarini: alimortarini@gmail.com

Financiamiento: Ninguno.

Conflicto de intereses: Ninguno que declarar.

Recibido: 1-6-2020

Aceptado: 30-9-2020

\section{INTRODUCCIÓN}

La pancreatitis recurrente (PR) ocurre en un 15-35\% en la edad pediátrica. Según el grupo INSPPIRE (por las siglas en inglés de International Study Group of Pediatric Pancreatitis), se define como 2 o más episodios distintos de pancreatitis aguda (PA) con resolución completa del dolor (por lo menos, 1 mes de intervalo entre los diagnósticos de PA) o normalización completa de enzimas pancreáticas (amilasa y lipasa) antes del diagnóstico del próximo episodio, junto con la resolución del dolor, independientemente del tiempo transcurrido entre episodios de PA.

La incidencia reportada es de 0,71 a $0,82 / 100000$, con una media de edad de presentación de 13 años. ${ }^{3}$ Existen dos tipos de pancreatitis autoinmune (PAI): el primero es más frecuente en Asia, y el segundo, en Europa y en Estados Unidos. ${ }^{4}$ Fue descrita inicialmente por Sarles, en 1961, como pancreatitis inflamatoria y luego pancreatitis esclerosante linfoplasmocitaria, y, finalmente, como PAI por Yoshida, en 1995. ${ }^{5}$

Si bien la PAI es una causa infrecuente dentro de las PR, es importante sospecharla e indagar antecedentes de autoinmunidad familiar para poder realizar un tratamiento oportuno. Se describe un caso clínico de una paciente que fue seguida en el Hospital Garrahan por PR, finalmente considerada PAI. Luego del tratamiento, se observó una mejoría en las imágenes y una disminución del número y de la gravedad de episodios de PA. Se considera también la importancia de un diagnóstico oportuno para prevenir lesiones crónicas e irreversibles en el páncreas.

\section{CASO CLÍNICO}

Paciente femenina de 7 años, con historia previa de 5 episodios totales de PA, uno de los cuales requirió manejo en Cuidados Intensivos. Antecedentes personales: convulsión febril medicada con fenobarbital desde los dos años, que fue suspendido luego del segundo episodio de pancreatitis. Autoinmunidad familiar de primer grado (tiroiditis de Hashimoto en el padre y abuela con hipotiroidismo).

En el primer episodio de PA, requirió 
internación en la Unidad de Terapia Intensiva Pediátrica (UTIP). En la ecografía abdominal, se evidenció líquido anecoico, sin litiasis vesicular, páncreas hipoecoico aumentado de tamaño con colección laminar en porción cefálica. Tomografía computada con derrame pleural bilateral, con aumento de líquido peripancreático, con aumento de tamaño y áreas de necrosis tras la administración de contraste. Se colocó nutrición parenteral y presentó buena evolución, con descenso progresivo de amilasa. Requirió internación por 9 días en la UTIP y 5 días en sala.

A los 2 meses del primer episodio, se reinternó por dolor abdominal intenso, vómitos alimentarios y análisis de laboratorio con hiperamilasemia (530 U/1) e hiperlipasemia (720 U /1). Se realizó una tomografía computada (TC) de abdomen, en la que se observó el páncreas aumentado de tamaño. Presentó resolución clínica con ayuno sin requerimientos de nutrición parenteral. Los siguientes episodios de pancreatitis fueron leves y mejoraron rápidamente con la suspensión de la vía oral.

Concurrió a nuestro Hospital, donde se realizaron los siguientes estudios: test del sudor y estudio molecular para fibrosis quística, lipidograma, prueba de Van de Kamer y elastasa, normales, anticuerpos para enfermedad celíaca negativos, anticuerpos antitiroideos positivos con hormonas periféricas normales, anticuerpos antimúsculo liso (ASMA) con especificidad actina y anti-nucleares (FAN) positivos, dosaje de IgG4 normal. En la colangiopancreatorresonancia se observó la vía biliar intra y extrahepática sin alteraciones, alteración del conducto de Wirsung sugestivo de PAI (Figura 1).

Inició tratamiento con meprednisona a razón de $1 \mathrm{mg} / \mathrm{kg} /$ día por 1 mes. Recibió, en total, tres meses de corticoides con descenso paulatino, resolución de la clínica y mejoría de las imágenes (Figura 2). En los primeros dos años luego de la suspensión de los corticoides, no presentó nuevos episodios. En el tercer año, requirió internación en dos oportunidades por dolor y elevación de amilasa y lipasa, que se resolvieron luego de 48 a 72 horas de ayuno. No presentó cambios significativos en las imágenes y mantuvo adecuado progreso pondoestatural (índice de masa corporal-IMC-: 18; peso/talla: del $115 \%$; superficie corporal: $1 \mathrm{~m}^{2}$ ) y suficiencia pancreática (elastasa normal).

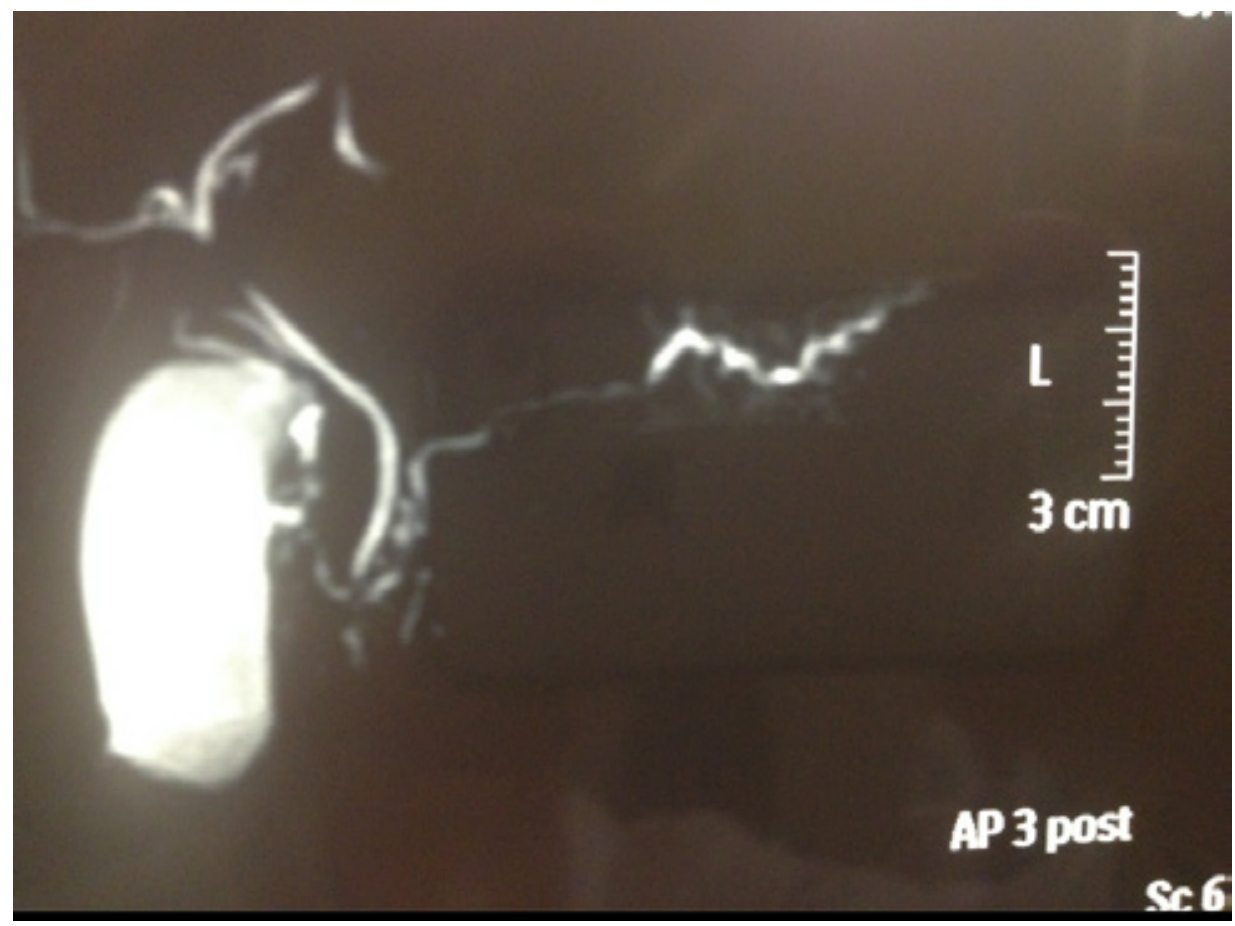

Colangiopancreatorresonancia: Conducto pancreático principal de calibre irregular, diámetro mayor de 2,6 mm a nivel del cuerpo y cola. 


\section{DISCUSIÓN}

La PAI es una causa infrecuente de PR, sobre todo, en pediatría. Se define como una enfermedad fibroinflamatoria sistémica que afecta al páncreas y a otros órganos, como los conductos biliares, las glándulas salivares, el retroperitoneo y los nódulos linfáticos, caracterizada por una buena respuesta clínica a los corticosteroides. ${ }^{4-6}$ Es una patología infrecuente, pero en auge en las últimas décadas, y es considerada como un tipo de pancreatitis crónica, con características histológicas, morfológicas y clínicas propias. Afecta a todas las razas y edades. En pediatría, es más frecuente en la adolescencia; presenta dolor abdominal, ictericia obstructiva y/o masa pancreática que podría simular cáncer pancreático.

En el análisis de laboratorio, es usual encontrar fosfatasa alcalina y transaminasas elevadas, mientras que amilasa y lipasa pueden ser normales al inicio de la PAI. ${ }^{6}$ Se presenta de forma aislada o en asociación a otras enfermedades autoinmunes, que pueden aparecer en cualquier momento de la enfermedad, particularmente, artritis reumatoide, hipotiroidismo, síndrome de Sjögren y enfermedad inflamatoria intestinal, además de cirrosis biliar primaria y pseudotumores inflamatorios. ${ }^{1,2}$

En la actualidad, se cuenta con criterios que facilitan el diagnóstico y tienen como principal objetivo excluir el de cáncer de páncreas. Afortunadamente en pediatría, a diferencia de los adultos, la principal sospecha ante un cuadro de ictericia obstructiva y masa pancreática es la PAI y no patología neoplásica del páncreas. La discriminación de PAI vs. cáncer de páncreas puede predecirse por los hallazgos que surgen de la ecoendoscopía, la TC y la colangiopancreatorresonancia. Sin embargo, la histología es obligatoria, aunque el riesgo de malignidad pancreática es muy bajo en los niños. En los adultos, el riesgo de desarrollar cáncer pancreático es 15 veces mayor que en la población general. $^{6}$

Según el Consenso Internacional de 2011, se describen dos tipos de PAI con diferentes formas clínicas, histopatológicas (que observan la presencia o ausencia de lesiones epiteliales granulocíticas), asociaciones (IgG4 y colitis ulcerosa) y evolución clínica. ${ }^{7-10}$ En pediatría, la mayoría corresponden al tipo 2, que ocurre con dosaje normal de IgG4 y es más frecuente en los jóvenes. Las causas de neoplasia pancreática en pediatría son raras.

Con respecto a los criterios diagnósticos, el Consenso Internacional de 2011 se basa en 5 características para el diagnóstico de PAI en el adulto: imágenes pancreáticas, serologías, manifestaciones extrapancreáticas (colangitis esclerosante, nefritis o masa renal, fibrosis retroperitoneal, masas o ganglios submandibulares), histología e inmunomarcación pancreática y la respuesta al tratamiento con corticoides. $^{8}$

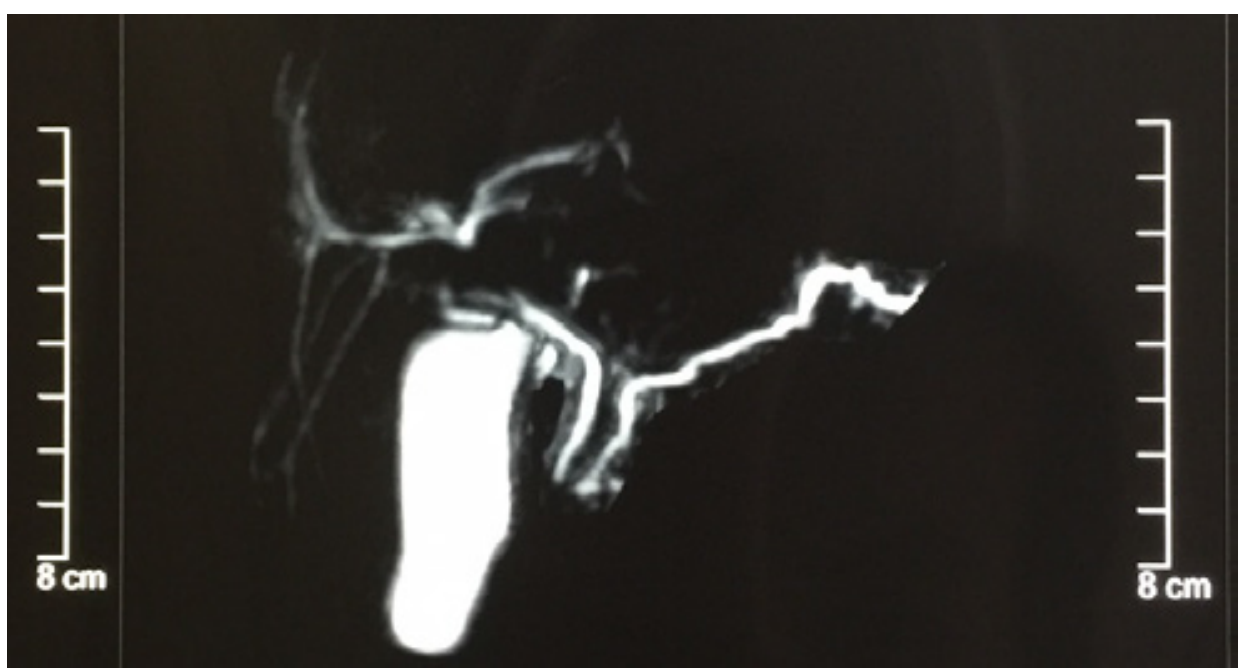

Colangiorresonancia postratamiento: Sin estenosis de conducto pancreático. 
En la PAI-1 o pancreatitis esclerosante linfoplasmocitaria, el compromiso extrapancreático es frecuente, y está asociada con enfermedades relacionadas con la IgG4. Mientras que la tipo 2 se limita a la enfermedad pancreática en sí, no asociada a IgG4. Es más común en los individuos jóvenes, caracterizada por el infiltrado de neutrófilos en el epitelio de los conductos pancreáticos, con buena respuesta a los corticoides. Se observaron pocos reportes con respecto a la recaída, a diferencia del tipo 1, en la que es frecuente. Si bien existe una clara relación con la enfermedad inflamatoria intestinal y la PAI-2, un $5 \%$ de los pacientes con PAI-1 también pueden desarrollarla. ${ }^{11}$

La mayoría de los pacientes pediátricos reportados en la literatura corresponden al tipo 2, que presenta dosaje normal de IgG4. Aquí se solicitó dosaje de IgG4 a los pacientes con PR sin otro diagnóstico etiológico. En nuestro caso, la paciente presentaba niveles de IgG4 normales y suficiencia pancreática. Fue considerada, finalmente, como PAI por hallazgos de la colangiopancreatorresonancia y los antecedentes de autoinmunidad personal y familiar, y presentó excelente respuesta clínica y evolución luego de recibir un ciclo de corticoides. Coincidiendo con la bibliografía, se observó una mejoría de las imágenes pancreáticas luego del tratamiento con corticoides y disminución del número y de la gravedad de los episodios.

Además, nuestra paciente presentó autoanticuerpos positivos sin etiología clara. Según los datos obtenidos por Pagliari, entre los estigmas inmunológicos definidos en niños con PAI, se ha demostrado la presencia de factor reumatoide en suero. La positividad de los anticuerpos antinucleares y el aumento de las inmunoglobulinas, en particular, de IgE, son considerados marcadores de baja especificidad para la PAI. Otro dato inmunológico fue el consumo del complemento C3 y C4 en un tercio de los pacientes, particularmente, en aquellos con altos niveles de complejo inmunitario circulante. ${ }^{6}$

Cabe resaltar que la IgG4 no es específica para PAI. Se ha visto elevada en enfermedades parasitarias, asma, dermatitis atópica, entre otras. Sin embargo, sí puede utilizarse para el seguimiento y el monitoreo de estos pacientes.

En cuanto al tratamiento, es de primera línea el uso de corticoides (de 1 a 1,5 mg/ kg/día con un máximo de 40-60 mg/ día) durante 2-4 semanas y luego se realiza un descenso paulatino. La introducción de un inmunomodulador, como 6-mercaptopurina, azatioprina, micofenolato o infliximab (si asocian enfermedad inflamatoria intestinal), es una alternativa para aquellos que requieran tratamiento de mantenimiento. ${ }^{11,12}$

La respuesta al tratamiento con corticoides se evalúa clínicamente a las 2 semanas y con ecografía y colangiopancreatorresonancia luego de 3 meses de inicio del tratamiento. La tasa de recaída es alta (del 30-40\%), y es mayor para la PAI-1. Se recomienda repetir un curso de esteroides seguido por una dosis baja de mantenimiento con prednisona. ${ }^{13,14}$ Nuestra paciente no presentó recaída hasta el momento.

Una de las limitantes en esta paciente fue la falta de estudio genético para el estudio de PR por no contar con dicho estudio. En la actualidad, hay insuficiente información para conocer las complicaciones a largo plazo, como insuficiencia pancreática exócrina y diabetes. Por lo tanto, los pacientes con PAI deberían ser controlados regularmente. ${ }^{12}$

\section{Agradecimiento}

A la Dra. Sandra Basso.

\section{REFERENCIAS}

1. Morinville VD, Husain SZ, Bai H, Bath B, et al. Definitions of pediatric pancreatitis and survey of present clinical practices. J Pediatr Gastroenterol Nutr. 2012; 55(3):261-5.

2. Uc A, Yen E, Wilschanski M, Werlin S, et al. Pediatric Acute Recurrent and Chronic Pancreatitis: Report From INSPPIRE Consortium. Gastroenterology. 2014; 146(5) Supp 1:S4.

3. Scheers I, Palermo JJ, Freedman S, Wilschanski M, et al. Autoimmune pancreatitis in children: characteristic features, diagnosis, and management. Am J Gastroenterol. 2017; 112(10):1604-11.

4. Okazaki K, Uchida K, Koyabu M, Miyoshi H, et al. Recent advances in the concept and diagnosis of autoimmune pancreatitis and IgG4-related disease. J Gastroenterol. 2011; 46(3):277-88.

5. Lariño Noia J, Iglesias García J, Domínguez Muñoz JE. Pancreatitis recurrente y crónica. Medicine. 2012; 11(8): 465-72.

6. Pagliari D, Rossella C, Rigante D. The Challenge of Autoimmune Pancreatitis: A Portrayal From the Pediatric Perspective. Pancreas. 2019; 48(5):605-12.

7. Ohara H, Nakazawa T, Ando T, Joh T. Systemic extrapancreatic lesions associated with autoimmune pancreatitis. J Gastroenterol. 2007; 42(Suppl 18):15-21.

8. Jackson Muñoz G. Pancreatitis Autoinmune. Rev Med Costa Rica Centroam. 2014; 71(613):765-71.

9. Galloway D, Wallihan D, Smith M, Abu-El-Haija M. An Unusual Presentation of Pediatric Autoimmune Pancreatitis. Pancreas. 2016; 45(2):e1-2.

10. Shimosegawa T, Chari ST, Frulloni L, Kamisawa T, et al. International consensus diagnostic criteria for autoimmune pancreatitis: guidelines of the International Association of Pancreatology. Pancreas. 2011; 40(3):352-8.

11. Bolia R, Chong SY, Coleman L, MacGregor D, et al. Autoimmune Pancreatitis and IgG4 Related Disease in Three Children. ACG Case Rep J. 2016; 3(4):e115. 
12. Scheers I, Palermo JJ, Freedman S, Wilschanski M, et al. Recommendations for Diagnosis and Management of Autoimmune Pancreatitis in Childhood: Consensus from INSPPIRE. J Pediatr Gastroenterol Nutr. 2018; 67(2):232-6.

13. Kubota K, Watanabe S, Uchiyama T, Kato S, et al. Factors predictive of relapse and spontaneous remission of autoimmune pancreatitis patients treated / not treated with corticosteroids. J Gastroenterol. 2011; 46(6):834-42.

14. Oshima A. Factors predictive of relapse / spontaneous remission in autoimmune pancreatitis patients on/not on corticos-teroid treatment. Sesión de carteles presentada en DDW 2011; mayo 7-10; Chicago, IL. Tu 193 